\title{
Motivação no ensino médio: Metas de realização e estratégias de aprendizagem
}

\author{
Acácia Aparecida A. dos Santos ${ }^{1}$ \\ iD http://orcid.org/0000-0002-8599-7465 \\ Amanda Lays M. Inácio ${ }^{1}$ \\ iD https://orcid.org/0000-0003-1892-6242
}

\begin{abstract}
Para citar este artigo: Santos, A. A. A., \& Inácio, A. L. M. (2020). Motivação no ensino médio: Metas de realização e estratégias de aprendizagem. Psicologia: Teoria e Prática, 22(2), 338-359.
\end{abstract}

Submissão: $12 / 04 / 2019$

Aceite: 15/04/2020

1 Universidade São Francisco (USF), Itatiba, SP, Brasil. 


\begin{abstract}
Resumo
Objetivou-se caracterizar o nível de motivação para aprender em termos de suas orientações a metas de realização e o emprego das estratégias de aprendizagem em alunos do ensino médio, bem como correlacionar as subescalas dos instrumentos e analisar diferenças considerando sexo e ano escolar. Buscou-se ainda identificar se a motivação para aprender pode predizer as estratégias de aprendizagem. Empregaram-se a Escala de Motivação para Aprendizagem e a Escala de Avaliação das Estratégias de Aprendizagem em 261 estudantes do ensino particular da Bahia, sendo a idade mínima 14 anos e a máxima $22(M=16,6, D P=1,4)$. Os resultados revelaram que os estudantes se mostraram mais orientados pela meta aprender e fazem uso das estratégias metacognitivas. A relação de predição indicou modelos explicativos com variância de $6 \%$ a $33 \%$. Sugere-se a continuidade das investigações a fim de se ampliar o entendimento acerca das relações existentes entre essas variáveis.

Palavras-chave: educação; ensino básico; estratégias de aprendizagem; metas de realização; motivação.
\end{abstract}

\title{
HIGH SCHOOL MOTIVATION: ACHIEVEMENT GOALS AND LEARNING STRATEGIES
}

\begin{abstract}
The objective was to characterize the level of motivation to learn in terms of its orientations to achievement goals and the use of learning strategies in high school students, as well as to correlate the subscales of the instruments and to analyze differences considering sex and school year. We also sought to identify whether the motivation to learn can predict learning strategies. The Learning Motivation Scale and the Learning Strategies Assessment Scale were used on 261 private school students in Bahia, with a minimum age of 14 years and a maximum of $22(M=16.6$, $\mathrm{SD}=1.4$ ). The results revealed that the students were more oriented towards the goal of learning and make use of metacognitive strategies. The prediction ratio indicated explanatory models with variance from $6 \%$ to $33 \%$. It is suggested that investigations continue to broaden the understanding of the relationships between these variables.
\end{abstract}

Keywords: education; basic education; learning strategies; achievement goals; motivation. 


\title{
MOTIVACIÓN DE LA ESCUELA SECUNDARIA: OBJETIVOS DE LOGRO Y ESTRATEGIAS DE APRENDIZAJE
}

\begin{abstract}
Resumen
El objetivo fue caracterizar el nivel de motivación para aprender en términos de sus orientaciones a los objetivos de logro y el uso de estrategias de aprendizaje en estudiantes de secundaria, así como correlacionar las subescalas de los instrumentos y analizar las diferencias considerando el sexo y el año escolar. También buscamos identificar si la motivación para aprender puede predecir estrategias de aprendizaje. La Escala de Motivación de Aprendizaje y la Escala de Evaluación de Estrategias de Aprendizaje se usaron en 261 estudiantes de escuelas privadas en Bahía, con una edad mínima de 14 años y un máximo de $22(M=16,6, D E=1,4)$. Los resultados revelaron que los estudiantes estaban más orientados hacia el objetivo de aprender y utilizar estrategias metacognitivas. El índice de predicción indicó modelos explicativos con una varianza del $6 \%$ al 33\%. Se sugiere continuar las investigaciones para ampliar la comprensión sobre las relaciones entre estas variables.

Palavras clave: educación; enseñanza básica; estrategias de aprendizaje; objetivos de logro; motivación.
\end{abstract}

\section{Introdução}

A aprendizagem no contexto escolar é mediada por fatores diversos que ultrapassam sobremaneira as capacidades cognitivas do estudante (Lemos et al., 2010). Entre esses fatores, encontra-se a motivação, que notadamente, no campo educacional, despertou em diversos pesquisadores a curiosidade de investigar as relações que se estabelecem entre esse construto e a aprendizagem (Alcará \& Santos, 2013; Albuquerque, Batista, Albuquerque, Oliveira, \& Pereira, 2016; Beluce \& Oliveira, 2016; Castro, Miranda, \& Leal, 2016; Elliot \& Murayama, 2008; Ferraz, Cantalice, \& Santos, 2019; Maieski, Oliveira, Beluce, \& Rufini, 2017; Oliveira, Santos, \& Inácio, 2017; Santos, Moraes, \& Lima, 2018; entre outros).

Em meio às principais teorias sociocognitivas que tratam da motivação para aprender, encontra-se a Teoria de Metas de Realizações (Elliott \& Dweck, 1988), que se concentra em explicar a orientação motivacional dos estudantes ante os processos acadêmicos. Assim, as metas estão diretamente relacionadas com a orientação do padrão de comportamento do indivíduo na realização das tarefas, sendo divididas em três componentes: meta aprender, meta performance-aproximação e meta performance-evitação. Essa organização foi a adotada como referencial do presente 
estudo, especialmente porque o instrumento de medida utilizado está embasado nesses três componentes. Entretanto, existe na literatura internacional uma organização em que as metas de realização se subdividem em quatro componentes, dois para a meta aprender e dois para a meta de execução, conforme referido por Elliot e McGregor (2001).

Na meta aprender, o estudante concentra-se no processo de aprendizado, no domínio do conteúdo e de realização de tarefa. Alunos orientados por essa meta se interessam mais pela absorção dos conteúdos, mantêm o interesse focado no desenvolvimento de habilidades e da criatividade e são propensos ao uso de estratégias mais adequadas para atingir seus objetivos (Bzuneck \& Boruchovitch, 2016). O aluno caracterizado pela meta performance-aproximação é apontado como aquele que sente necessidade de se destacar entre os colegas, mostrando-se mais inteligente, sem que o seu foco primordial esteja na aprendizagem efetiva. Aqueles orientados pela meta performance-evitação objetivam salvaguardar-se da desvalorização por parte de professores e colegas, preocupando-se, primordialmente, em não parecerem incompetentes (Senko \& Hulleman, 2013; Zenorini \& Santos, 2010).

Quando se focalizam as metas de realização, outro construto que aparece de modo recorrente diz respeito às estratégias de aprendizagem. A literatura tem apontado que o uso das estratégias de aprendizagem encontra-se condicionado a uma série de fatores, muitos deles de natureza motivacional (Alcará \& Santos, 2013; Greene, Miller, Crowson, Duke, \& Akey, 2004; Perassinoto, Boruchovitch, \& Bzuneck, 2013). Conforme destacam Zenorini e Santos (2010), o emprego de estratégias no momento de aprender é uma tarefa que exige esforço e persistência e que, portanto, requer um padrão motivacional adequado. Assim, o aluno pode ter domínio das estratégias e não as utilizar se não estiver motivado para as atividades.

As estratégias de aprendizagem são definidas como ações mentais e comportamentos com os quais o aluno se envolve durante a aprendizagem e que facilitam a recuperação de conhecimentos já adquiridos (Dembo, 1994; Weinstein \& Mayer, 1983). Essas estratégias são importantes porque fornecem suporte essencial para a aquisição de satisfatório desempenho acadêmico (Prates, Lima, \& Ciasca, 2016). O uso de estratégias possibilita o eficiente armazenamento da informação e sua eficaz recuperação, e, para tanto, é importante que o estudante tenha conhecimento delas e saiba como utilizá-las adequadamente (Lima \& Santos, 2016; Oliveira et al., 2017). 
As estratégias podem ser analisadas segundo duas vertentes: as cognitivas e as metacognitivas. As estratégias cognitivas consistem em organizar, elaborar e integrar a informação, enquanto as metacognitivas envolvem o planejamento, o monitoramento, a regulação do próprio pensamento e a manutenção de um estado interno satisfatório à aprendizagem do indivíduo. Assim, relacionam-se à maneira como o indivíduo percebe analiticamente as partes para entender o todo. As estratégias metacognitivas apresentam maior grau de complexidade, pois se relacionam com a tomada de consciência necessária para a execução de uma tarefa e com a capacidade de pensar sobre os próprios pensamentos, envolvendo planejamento, regulação, controle e execução do processo (Dembo, 1994; Perassinoto et al., 2013; Suehiro, Boruchovitch, \& Schelini, 2018).

Pesquisas nacionais que focalizam, simultaneamente, a motivação para aprender e as estratégias de aprendizagem ainda são escassas, sobretudo no ensino médio. No sistema educacional brasileiro, é consensual a percepção de que essa etapa de ensino possui importantes contradições, considerando seus problemas de acesso e permanência, a qualidade da educação ofertada e, ainda, questões referentes à sua identidade, que se mostram, entre outras formas, por meio dessa escassez (Ferreira \& Silva, 2017; Inácio, 2018; Krawczyk, 2011).

Os estudos que abordam a temática concentram-se em outras etapas de ensino, como o ensino fundamental (Costa \& Boruchovitch, 2015; Ferraz et al., 2019; Lima \& Santos, 2016; Maieski et al., 2017; Perassinoto et al., 2013; Prates et al., 2016; Santos et al., 2018) e ensino superior (Alcará \& Santos, 2013; Albuquerque et al., 2016; Beluce \& Oliveira, 2016; Castro et al., 2016). Contudo, destaca-se que tais estudos fizeram uso de diferentes instrumentos para avaliação desses construtos, o que dificulta comparações por serem fundamentados em perspectivas teóricas muitas vezes distintas.

A título de exemplo, no estudo de Perassinoto et al. (2013), realizado com 314 estudantes do terceiro ao nono ano do ensino fundamental público do interior de São Paulo, de ambos os sexos, foram encontradas correlações positivas e estatisticamente significativas entre as estratégias de aprendizagem e a motivação. No entanto, esta última fora avaliada com base na Teoria da Autodeterminação, que analisa os aspectos motivacionais intrínsecos e extrínsecos dos indivíduos.

Em razão da escassez de pesquisas que abarquem simultaneamente as estratégias de aprendizagem e a motivação para aprender e as diferenciações entre 
as perspectivas teóricas, buscou-se verificar estudos que abrangessem ao menos uma das variáveis em questão. Com essa proposta, serão apresentadas, na sequência, as pesquisas de Santos et al. (2018) com a motivação para aprender e de Scacchetti, Oliveira e Moreira (2015) e Inácio (2018) acerca das estratégias de aprendizagem.

Com a proposta de identificar a relação existente entre compreensão de leitura, avaliada pelo teste de Cloze, e a motivação para aprender, com a Escala de Motivação para Aprendizagem (EMAPRE), Santos et al. (2018) avaliaram 169 estudantes do sexto ao nono ano do ensino fundamental público do estado de São Paulo, de ambos os sexos, com idades entre 11 e 17 anos ( $M=12,7$ anos, DP = 1,2). Os resultados indicaram que a meta aprender foi a mais empregada, havendo uma correlação positiva e significativa entre ela e os escores do Cloze. Houve correlação negativa e significativa no que diz respeito às metas performance-aproximação e evitação com a meta aprender.

As análises do estudo de Santos et al. (2018) indicaram também a existência de diferença significativa entre os sexos, que ocorreu somente em relação à meta performance-aproximação ( $t=2,389 ; p=0,018)$, em que a pontuação dos meninos foi significativamente maior do que a das meninas. Quanto aos anos escolares, houve diferença na meta performance-aproximação ( $F=4,095 ; p=0,008)$, em que os alunos do sexto ano sobressaíram em relação aos demais.

A fim de verificar evidências de validade de um instrumento, resultante da adaptação da Escala de Avaliação das Estratégias de Aprendizagem para o Ensino Fundamental (EAVAP-EF), Scacchetti et al. (2015) trabalharam com uma amostra de 709 estudantes do ensino técnico-profissional, provenientes de duas instituições do Paraná, de ambos os sexos. A análise fatorial exploratória indicou uma estrutura de três fatores, conforme previsto teoricamente. Os estudantes pontuaram em nível mais elevado na ausência de estratégias metacognitivas disfuncionais, sendo a menor pontuação nas estratégias metacognitivas. Entretanto, tais resultados devem ser vistos com cautela, haja vista que a consistência interna das subescalas do instrumento, avaliada por meio do alfa de Cronbach, não apresentou índices considerados aceitáveis em todas as subescalas ( $\alpha=0,57$ nas estratégias metacognitivas).

Mesmo com essa ressalva ao instrumento, Inácio (2018) utilizou a EAVAP-EF com 12 itens adaptados de Schacchetti et al. (2015). Seu intuito foi analisar as 
diferenças entre os estilos intelectuais, as estratégias de aprendizagem, a compreensão de leitura e o desempenho escolar em 764 estudantes do primeiro ao terceiro ano do ensino médio, oriundos de três escolas no estado do Paraná, uma pública e duas particulares, de ambos os sexos. Os resultados demonstraram que os estudantes fazem mais uso das estratégias metacognitivas no momento de aprender, o que também ocorre em relação às modalidades de ensinos público e particular.

Considerando a relevância desses construtos para a avaliação psicoeducacional na busca de indicadores que auxiliem no desenvolvimento do sucesso escolar dos estudantes do ensino médio (Bzuneck \& Boruchovitch, 2016; Greene et al., 2004; Inácio, 2018), a presente pesquisa teve como objetivos: 1. caracterizar o nível de motivação para a realização das atividades escolares e identificar em que medida os alunos relatam utilizar estratégias de aprendizagem; 2. correlacionar as subescalas de motivação para aprender e as estratégias de aprendizagem; 3. analisar possíveis diferenças nas pontuações das escalas considerando o sexo e o ano escolar; 4. identificar se a motivação para aprender pode predizer o emprego das estratégias de aprendizagem.

\section{Método}

\subsection{Participantes}

A amostra escolhida por conveniência foi composta por 261 estudantes do ensino médio de uma instituição particular do interior do estado da Bahia. Desse total, $52,1 \%(n=136)$ se declararam do sexo feminino e $44,9 \%(n=125)$ do masculino. A média de idade foi de 16 anos e 6 meses ( $D P=1,4$ ), sendo 14 a idade mínima e a máxima 22 anos. Quanto ao ano escolar, 32,2\% ( $n=84$ ) estavam matriculados no primeiro ano, $27,6 \%(n=72)$ no segundo e $40,2 \%(n=105)$ no terceiro.

\subsection{Instrumentos}

- Escala de Motivação para Aprendizagem - EMAPRE (Zenorini \& Santos, 2010): composta de 28 itens distribuídos em três fatores, sendo 12 da subescala da meta aprender, nove da meta performance-aproximação e sete da meta performance-evitação. É estruturada em escala do tipo Likert com três opções de resposta: discordo - um ponto; não sei - dois pontos; e concordo 
- três pontos. Um exemplo de item seria: "8) Na minha turma, eu quero me sair melhor que os demais". A EMAPRE foi submetida à análise de consistência interna dos itens, medida pelo alfa de Cronbach ( $\alpha$ ). A escala total apresentou alfa de $0,73,0,80$ de meta aprender, 0,76 de meta performance-aproximação e 0,73 de meta performance-evitação.

- Escala de Avaliação das Estratégias de Aprendizagem para o Ensino Fundamental - EAVAP-EF (Oliveira, Boruchovitch, \& Santos, 2010): o instrumento possui 31 itens e visa identificar as estratégias cognitivas e metacognitivas, bem como a ausência de estratégias metacognitivas disfuncionais utilizadas pelos estudantes. A escala é do tipo Likert de três pontos, sendo: sempre (dois pontos), às vezes (um ponto) e nunca (zero ponto), com pontuação invertida nas questões da subescala ausência de estratégias metacognitivas disfuncionais. Possui evidências de validade de conteúdo, de critério concorrente e preditivo. Quanto à fidedignidade, o alfa de Cronbach da escala total foi de $0,79,0,80$ na subescala ausência de estratégias de aprendizagem, 0,74 de estratégias cognitivas e 0,62 de estratégias metacognitivas. Decidiu-se pelo emprego da escala com propriedades psicométricas para o ensino fundamental após análise da semântica dos itens, em que foi identificado que estes não possuíam um vocabulário tão distinto em relação ao ensino médio. Por exemplo, item 8 "Na minha turma eu quero me sair meIhor que os demais" e item 21 "Gosto quando uma matéria me faz sentir vontade de aprender mais". Além disso, de acordo com os autores da EAVAP-EF (Oliveira et al., 2010), a escala pretende avaliar um público de 7 a 16 anos, o que corresponderia, em parte, à faixa etária do ensino médio.

\subsection{Procedimentos}

A pesquisa se respaldou na Resolução n. 466/2012 do Conselho Nacional de Saúde e em seus complementares, sendo registrada e aprovada pelo Comitê de Ética em Pesquisa (CEP) de uma universidade pública, sob o Parecer n. 1.748.266. A coleta de dados ocorreu de forma coletiva em sala de aula, na seguinte ordem: EMAPRE e EAVAP-EF, com tempo médio de aplicação para os dois instrumentos de 45 minutos. Participaram do estudo somente os estudantes que devolveram assinado pelos responsáveis o Termo de Consentimento Livre e Esclarecido (TCLE). 


\subsection{Análise de dados}

Os dados foram organizados em planilha eletrônica, e adotaram-se como método de análise as estatísticas descritiva e inferencial realizadas por meio do Programa IBM - Statistical Package for the Social Sciences for Windows versão 22.0 - SPSS. Análises descritivas foram empregadas para verificar os resultados de média e o desvio padrão referente aos dados dos participantes e das pontuações nos instrumentos. Em vias de encontrar as correlações entre os dois instrumentos, utilizou-se a análise de correlação $r$ de Pearson com interpretação das magnitudes segundo Cohen (1988). Aplicou-se o teste t de Student para analisar possíveis diferenças nas pontuações das escalas considerando o sexo dos estudantes. A análise de variância ANOVA foi utilizada a fim de examinar as diferenças existentes quanto ao ano escolar, sendo solicitado o teste post hoc de Tukey para a separação de subgrupos de desempenho e adotado nível de significância de 0,05. Nesse caso, aplicou-se a análise do d de Cohen para determinar o significado do efeito estatístico no teste t. Por fim, empregaram-se quatro análises de regressão pelo método Stepwise, sendo os fatores da subescala EAVAP-EF inseridos como variável dependente, e as variáveis preditoras foram os fatores da motivação para aprender.

\section{Resultados}

Os resultados serão apresentados na sequência, conforme a descrição dos objetivos do presente estudo. No que se refere à averiguação das estratégias de aprendizagem e motivação para aprender, na Tabela 3.1 podem ser observadas as pontuações das médias, do desvio padrão e da média ponderada das subescalas. Ressalta-se que a média ponderada se refere à divisão do total de cada subescala pelo número de itens. Assim, pode-se obter o resultado de predominância, já que os resultados serão comparados de maneira semelhante. 
Tabela 3.1. Estatísticas descritivas da motivação para aprender e das estratégias de aprendizagem.

\begin{tabular}{lccc}
\hline & Médias & $\begin{array}{c}\text { Desvio } \\
\text { padrão }\end{array}$ & $\begin{array}{c}\text { Média } \\
\text { ponderada }\end{array}$ \\
\hline Meta aprender & 28,85 & 4,70 & 2,40 \\
Meta performance-aproximação & 15,99 & 4,19 & 1,77 \\
Meta performance-evitação & 10,20 & 3,36 & 1,45 \\
Motivação Total & 55,03 & 6,85 & 1,77 \\
Ausência de estratégias metacognitivas & 13,77 & 3,95 & 1,05 \\
disfuncionais & 10,92 & 4,34 & 0,99 \\
Estratégias cognitivas & 11,17 & 1,90 & 1,59 \\
Estratégias metacognitivas & 35,85 & 7,23 & 1,28 \\
Estratégias total & & & \\
\hline
\end{tabular}

Com base na Tabela 3.1, pode-se observar que os estudantes se encontram mais orientados pela meta aprender. Quanto às estratégias utilizadas, eles fazem mais uso de estratégias metacognitivas no momento da aprendizagem, seguido pela ausência de estratégias metacognitivas disfuncionais.

Com a finalidade de analisar as relações existentes entre as subescalas de motivação para aprender e as estratégias de aprendizagem, recorreu-se à correlação de Pearson. Os resultados da análise podem ser vistos na Tabela 3.2.

Tabela 3.2. Correlações entre a EMAPRE e a EAVAP-EF.

\begin{tabular}{lcccc}
\hline & $\begin{array}{c}\text { Ausência de } \\
\text { estratégias } \\
\text { meta } \\
\text { disfuncionais }\end{array}$ & $\begin{array}{c}\text { Estratégias } \\
\text { cognitivas }\end{array}$ & $\begin{array}{c}\text { Estratégias } \\
\text { metacognitivas }\end{array}$ & $\begin{array}{c}\text { Estratégias } \\
\text { total }\end{array}$ \\
\hline $\begin{array}{l}\text { Meta aprender } \\
\text { Meta performance- }\end{array}$ & $0,317^{* *}$ & $0,492^{* *}$ & $0,265^{* *}$ & $0,538^{* *}$ \\
$\begin{array}{l}\text { aproximação } \\
\text { Meta performance- } \\
\text { evitação }\end{array}$ & $-0,116^{*}$ & $0,139^{*}$ & 0,018 & 0,025 \\
Motivação total & $-0,417^{* *}$ & $-0,168^{* *}$ & $-0,127^{*}$ & $-0,362^{* *}$ \\
\hline
\end{tabular}

Nível de significância: $* p<0,05 ; * p<0,01$. 
Tal como demonstrado na Tabela 3.2, observa-se que houve correlações positivas e significativas entre a meta aprender e todos os fatores da EAVAP-EF, com magnitudes do efeito entre pequenas e médias segundo a classificação de Cohen (1988). Ademais, todas as correlações entre a meta performance-evitação e a EAVAP-EF foram negativas e significativas, com magnitudes entre pequenas e médias. A meta performance-aproximação se correlacionou negativa e significativamente com a ausência de estratégias disfuncionais (magnitude pequena) e positivamente com as estratégias cognitivas (magnitude pequena). Por fim, houve correlação positiva e significativa entre a motivação total e as estratégias cognitivas, sendo a magnitude considerada média e entre a motivação total e as estratégias no total, sendo esta última de magnitude pequena.

Na sequência, foram averiguadas as diferenças em razão do sexo dos participantes. Os resultados são apresentados na Tabela 3.3.

Tabela 3.3. Diferenças em razão do sexo.

\begin{tabular}{lcccccc}
\hline Subescalas & Sexo & Média & DP & $t$ & $\boldsymbol{p}$ & $\boldsymbol{d}$ \\
\hline Meta aprender & Masculino & 28,10 & 4,992 & $-2,481$ & 0,014 & $-0,308$ \\
& Feminino & 29,54 & 4,334 & & & \\
Meta performance- & Masculino & 16,90 & 4,515 & & & \\
aproximação & Feminino & 15,15 & 3,702 & 3,419 & 0,001 & 0,424 \\
Estratégias & Masculino & 9,75 & 4,334 & $-4,289$ & 0,001 & $-0,532$ \\
cognitivas & Feminino & 11,99 & 4,077 & & & \\
Estratégias & Masculino & 10,85 & 2,114 & $-2,643$ & 0,009 & $-0,329$ \\
metacognitivas & Feminino & 11,46 & 1,633 & & & \\
Estratégias & Masculino & 33,91 & 7,241 & $-4,4288$ & 0,001 & $-0,530$ \\
total & Feminino & 37,63 & 6,775 & & & \\
\hline
\end{tabular}

De acordo com os resultados evidenciados na Tabela 3.3 é possível identificar que o sexo masculino obteve pontuação mais elevada apenas na subescala da meta performance-aproximação da Escala de Motivação para Aprender. Ademais, o sexo feminino obteve pontuações mais altas nas subescalas da meta aprender, estratégias cognitivas, estratégias metacognitivas e estratégias no total. A análise do d de Cohen indicou efeito estatístico médio em todas as subescalas analisadas. 
Ressalta-se que um dos objetivos do presente estudo era buscar diferenças em razão do ano escolar, contudo, elas ocorreram ao acaso.

O último objetivo do presente estudo foi identificar se a motivação para aprender pode predizer o emprego das estratégias de aprendizagem. Para tanto, realizaram-se quatro análises de regressão com os fatores da subescala EAVAP-EF inseridos como variável dependente, e as variáveis preditoras foram os fatores da motivação para aprender. A Tabela 3.4 apresenta os resultados encontrados.

Tabela 3.4. Regressão dos fatores da motivação para aprender.

\begin{tabular}{|c|c|c|c|c|c|}
\hline \multicolumn{2}{|c|}{ Variáveis dependentes } & $\begin{array}{c}\text { Coeficiente } \\
\text { padronizado }\end{array}$ & \multirow{2}{*}{$\mathbf{T}$} & \multirow{2}{*}{$p$} & \multirow{2}{*}{$\mathbf{R}^{2}$ ajustado } \\
\hline $\begin{array}{c}\text { Ausência } \\
\text { metacognitiv }\end{array}$ & $\begin{array}{l}\text { tratégias } \\
\text { isfuncionais }\end{array}$ & $B$ & & & \\
\hline \multirow{3}{*}{ Modelo 3} & Meta PE & $-0,35$ & $-6,19$ & $<0,01$ & \multirow{3}{*}{0,22} \\
\hline & $\begin{array}{c}\text { Meta } \\
\text { aprender }\end{array}$ & 0,23 & 4,13 & $<0,01$ & \\
\hline & Meta PA & $-0,11$ & $-2,09$ & $<0,01$ & \\
\hline \multirow{2}{*}{\multicolumn{2}{|c|}{ Estratégias cognitivas }} & $\begin{array}{c}\text { Coeficiente } \\
\text { padronizado }\end{array}$ & \multirow[t]{2}{*}{$\mathbf{T}$} & \multirow[t]{2}{*}{$p$} & \multirow[t]{2}{*}{$\mathrm{R}^{2}$ ajustado } \\
\hline & & $B$ & & & \\
\hline & $\begin{array}{c}\text { Meta } \\
\text { aprender }\end{array}$ & 0,49 & 9,08 & $<0,01$ & 0,23 \\
\hline \multirow{2}{*}{\multicolumn{2}{|c|}{ Estratégias metacognitivas }} & $\begin{array}{c}\text { Coeficiente } \\
\text { padronizado }\end{array}$ & \multirow[t]{2}{*}{$\mathbf{T}$} & \multirow[t]{2}{*}{$p$} & \multirow[t]{2}{*}{$\mathbf{R}^{2}$ ajustado } \\
\hline & & $B$ & & & \\
\hline Modelo 1 & $\begin{array}{c}\text { Meta } \\
\text { aprender }\end{array}$ & 0,26 & 4,42 & $<0,01$ & 0,06 \\
\hline \multirow{2}{*}{\multicolumn{2}{|c|}{ Estratégias total }} & $\begin{array}{c}\text { Coeficiente } \\
\text { padronizado }\end{array}$ & \multirow[t]{2}{*}{$\mathbf{T}$} & \multirow[t]{2}{*}{$p$} & \multirow[t]{2}{*}{$\mathbf{R}^{2}$ ajustado } \\
\hline & & $B$ & & & \\
\hline \multirow[t]{2}{*}{ Modelo 2} & $\begin{array}{c}\text { Meta } \\
\text { aprender }\end{array}$ & 0,47 & 9,12 & $<0,01$ & \multirow[t]{2}{*}{0,33} \\
\hline & Meta PE & $-0,23$ & $-4,58$ & $<0,01$ & \\
\hline
\end{tabular}

Nota: Meta PE = performance-evitação; meta PA = performance-aproximação. 
A primeira análise teve como variável dependente a ausência de estratégias metacognitivas disfuncionais e resultou em três modelos estatisticamente significativos, e o terceiro foi o que melhor explicou a variância $\left(R^{2}\right.$ ajustado $\left.=0,22\right)$. Assim, as metas performance-evitação, aprender e performance-aproximação são preditoras da ausência de estratégias metacognitivas disfuncionais.

A subescala de estratégias cognitivas foi a variável dependente da segunda análise de regressão, sendo apresentado somente um modelo explicativo estatisticamente significativo ( $R^{2}$ ajustado $\left.=0,23\right)$. Com base nisso, a meta aprender é preditora das estratégias cognitivas. Na sequência, a terceira análise teve como variável dependente as estratégias metacognitivas, sendo apresentado também um modelo explicativo estatisticamente significativo $\left(R^{2}\right.$ ajustado $\left.=0,06\right)$. Desse modo, a meta aprender pode ser considerada preditora das estratégias metacognitivas.

Por fim, a quarta análise teve como variável dependente a escala total de estratégias de aprendizagem e indicou dois modelos estatisticamente significativos, e o segundo modelo foi o que melhor explicou a variância $\left(R^{2}\right.$ ajustado $=0,33$ ). Assim, as metas aprender e performance-evitação são preditoras das estratégias de aprendizagem.

\section{Discussão}

As análises descritivas dos instrumentos indicaram que os estudantes se encontram mais orientados pela meta aprender, o que significa dizer que eles são mais criativos e interessados na absorção dos conteúdos, além de fazerem uso de estratégias mais adequadas no momento da aprendizagem (Bzuneck \& Boruchovitch, 2016; Senko \& Hulleman, 2013). Diante do exposto, pode-se supor que, ao longo das etapas escolares, os alunos tendem a orientar-se mais para o significado efetivo do conhecimento do que pela evitação de situações desagradáveis envolvidas na aprendizagem, tal como parecer incompetente perante os colegas.

Esse quadro é benéfico ao contexto educacional, haja vista que a compreensão do real significado dos objetivos de aprendizagem contribui sobremaneira para a aquisição dos conhecimentos (Alcará \& Santos, 2013; Bzuneck \& Boruchovitch, 2016). Ademais, esses resultados indicam que, mesmo com as dificuldades historicamente arraigadas ao ensino médio no Brasil, no que se refere tanto à organização pedagógico-curricular quanto à falta de investimento da formação continuada dos docentes, entre outros (Ferreira \& Silva, 2017; Krawczyk, 2011), os estudantes têm 
conseguindo lidar com as adversidades e se orientar efetivamente pela importância do aprendizado das atividades escolares.

O resultado que evidenciou a prevalência do uso de estratégias metacognitivas no momento da aprendizagem se mostra bastante relevante ao contexto educacional. Isso indica que os estudantes possuem a tomada de consciência necessária para refletir sobre o próprio pensamento, planejar, regular e controlar seu comportamento em direção à aprendizagem, tarefa que exige um alto grau de complexidade (Dembo, 1994; Oliveira et al., 2017). Ademais, o emprego dessas estratégias é concebido na literatura como um suporte essencial para um desempenho acadêmico satisfatório (Lima \& Santos, 2016; Prates et al., 2016).

Esse resultado mostra-se consonante com os do estudo de Inácio (2018) realizado com o ensino médio público e particular. Contudo, difere dos achados de Scacchetti et al. (2015) feito com estudantes do ensino técnico-profissional, em que a menor pontuação foi em relação às estratégias metacognitivas. Essas divergências revelam a necessidade de investigações mais aprofundadas, sendo importante considerar também o fato de os estudos citados serem decorrentes de amostras com graus de escolaridade distintos, a saber, ensino médio e ensino técnico-profissional.

Os resultados tornam-se ainda mais relevantes quando se analisa o ensino médio como etapa que, posteriormente, levará o aluno ao ingresso no ensino superior e/ou ao mercado de trabalho. Tais dados possibilitam elucidar que os estudantes do ensino médio fazem uso das ferramentas necessárias para que a aprendizagem ocorra de modo satisfatório, compreendendo a relevância dos conhecimentos adquiridos não somente para a área acadêmica, mas também para sua formação integral como agentes que integram a sociedade, na qual atuarão exercendo suas profissões futuras (Alcará \& Santos, 2013; Inácio, 2018).

De modo análogo, cabe destacar que as diferenças educacionais nas diversas regiões do Brasil, bem como as desigualdades sociais do país, também se revelam como um ponto cabível de discussão, pois a garantia à universalização do ensino e a obrigatoriedade do ensino médio, como etapa final da educação básica, não asseguram o sucesso acadêmico e as mesmas oportunidades a todos os estudantes (Ferreira \& Silva, 2017; Inácio, 2018). As análises psicoeducacionais empregadas possibilitam problematizações teóricas quanto à importância da motivação para aprender e do uso de estratégias de aprendizagem para a aquisição de conhecimentos. Também permitem refletir sobre os aspectos deficitários encontrados no 
processo educacional brasileiro, a fim de que eles possam ser superados. Ademais, autorizam a pensar em práticas passíveis de ser implementadas, tais como a meIhor forma de ensinar aos alunos o uso de estratégias e a importância da formação continuada dos docentes.

Em face do segundo objetivo, de correlacionar as subescalas de motivação e estratégias de aprendizagem, os resultados evidenciaram que houve correlação entre a meta aprender e todas as subescalas de estratégias de aprendizagem. Não foram encontradas pesquisas que empregassem os mesmos instrumentos, embora os resultados do estudo de Castro et al. (2016) tenham se mostrado bastante semelhantes. Isso ocorreu porque, nesse estudo, a motivação intrínseca, subescala que mais se aproxima da meta aprender e faz parte da Teoria da Autodeterminação, correlacionou-se com todas as subescalas de estratégias (avaliadas pela EAVAP-EF, assim como no presente estudo).

Correlações entre esses construtos eram esperadas, tendo em vista a contribuição da motivação para o emprego de estratégias de aprendizagem mais adequadas no momento do estudo, conforme já evidenciado por outros autores (Alcará \& Santos, 2013; Greene et al., 2004; Zenorini \& Santos, 2010). Vale destacar ainda, conforme Greene et al. (2004), o quanto a motivação influencia no desenvolvimento cognitivo e, consequentemente, em resultados acadêmicos mais satisfatórios. Desse modo, o esforço empreendido nas tarefas, a persistência diante das dificuldades e as metas previamente estabelecidas irão depender, entre outros aspectos, dos níveis motivacionais do estudante.

Além disso, houve correlações negativas e significativas entre a meta performance-evitação e as subescalas de estratégias de aprendizagem. Não foram encontrados estudos que corroborassem tais resultados, contudo tem-se que esta pesquisa faz sentido à medida que a meta em questão diz respeito a características como preocupação do estudante em não parecer incompetente perante os demais, o que não reflete uma responsabilidade com a aprendizagem em si, corroborando um menor emprego de estratégias que auxiliem a aquisição dos conhecimentos (Bzuneck \& Boruchovitch, 2016).

Atendendo ao terceiro objetivo, foi realizada a análise de possíveis diferenças nas pontuações das escalas considerando o sexo e o ano escolar. Quanto ao sexo, essas diferenças foram significativas nas subescalas das metas aprender e performance-aproximação, e o sexo masculino obteve pontuação mais elevada so- 
mente na última subescala. Houve diferença significativa também em estratégias cognitivas, metacognitivas e estratégias total, todas com pontuação mais alta em relação ao sexo feminino.

Essa verificação é consonante com os achados de Santos et al. (2018), que mostraram diferenças significativas em relação à meta performance-aproximação com pontuação dos meninos maior do que a das meninas. Cabe ressaltar que essa meta se refere a indivíduos com necessidade de se destacar entre os colegas, mostrando-se mais inteligente, sem que o seu foco primordial esteja efetivamente na aprendizagem (Bzuneck \& Boruchovitch, 2016; Zenorini \& Santos, 2010).

A análise dessas diferenças foi empregada em vias de possibilitar indicadores em face das controvérsias presentes na literatura sobre a medida de avaliação do sexo, que se mostra bastante heterogênea. As diferenças encontradas podem decorrer de fatores culturais, sociais, escolares e inclusive ao acaso, evidenciando a importância de que a variável em questão continue a ser investigada (Dal'Igna, 2007). Uma explicação que permita a leitura dos dados obtidos pode ser encontrada no fator cultural que impele às mulheres uma exigência maior de êxito nos diversos âmbitos cotidianos, inclusive no desempenho educacional.

Destaca-se ainda que não foram encontradas diferenças em decorrência do ano escolar. Contudo, algumas pesquisas com alunos do ensino fundamental evidenciam que os estudantes se encontram mais motivados para aprender no início da escolarização (Ferraz et al., 2019; Santos et al., 2018). Expectativas similares existem em relação às estratégias de aprendizagem (Oliveira, Boruchovitch, \& Santos, 2011). Diante disso, formulou-se a hipótese de que haveria diferenças também no ensino médio, o que não foi corroborado.

Pressupõe-se que tal fato possa ter ocorrido em virtude da especificidade da amostra empregada no presente estudo, por se tratar de uma única instituição particular do interior do estado da Bahia. Contudo, nas pesquisas anteriormente citadas (Ferraz et al., 2019; Oliveira et al., 2011; Santos et al., 2018), em que se verificou a diminuição da motivação para aprender e do emprego de estratégias de aprendizagem ao longo dos anos escolares, levantou-se a hipótese de que essa redução poderia estar relacionada a diversos fatores. Sobre isso, Oliveira et al. (2017) relatam que muitas das defasagens encontradas em alunos do ensino médio são decorrentes de lacunas presentes em etapas anteriores durante o ensino básico, o que evidencia a necessidade de que os professores promovam processos 
de ensino e aprendizagem mais efetivos para o aluno, ao longo de todo o processo escolar.

Por fim, buscou-se identificar se a motivação para aprender poderia predizer o emprego das estratégias de aprendizagem. A hipótese era de que essa relação de predição ocorreria, tendo em vista as correlações existentes entre a meta aprender e as subescalas de estratégias e, ainda, algumas evidências da literatura (Perassinoto et al., 2013; Zenorini \& Santos, 2010). Os estudantes, quando orientados à meta aprender, envolvem-se com o processo de aprendizagem, buscam novos conhecimentos e aplicam esforço nas tarefas acadêmicas, obtendo maior autocontrole e autonomia em suas atividades. Como consequência, utilizam mais adequadamente as estratégias de aprendizagem (Alcará \& Santos, 2013)

Realizaram-se quatro análises de regressão com os fatores da subescala EAVAP-EF inseridos como variável dependente, e as variáveis preditoras foram os fatores da motivação para aprender. Assim, as metas performance-evitação, aprender e performance-aproximação foram preditoras da ausência de estratégias metacognitivas disfuncionais. Esse resultado sugere que a aprendizagem pela necessidade de se destacar entre os colegas e/ou para salvaguarda-se de desvalorização, bem como comportamentos como concentrar-se no processo de aprendizado estando efetivamente interessado no conteúdo (Bzuneck \& Boruchovitch, 2016; Zenorini \& Santos, 2010) podem predizer a ausência do emprego de estratégias que venham a prejudicar a aquisição do conhecimento.

A meta aprender mostrou-se preditora das estratégias cognitivas. Com base nisso, pode-se afirmar que a atitude mais voltada ao domínio do conteúdo com único propósito no aprendizado efetivo (Senko \& Hulleman, 2013) é capaz de predizer comportamentos como organizar, elaborar e integrar a informações (Dembo, 1994, Suehiro et al., 2018). Na sequência, a meta aprender mostrou-se preditora das estratégias metacognitivas. Desse modo, pressupõe-se que, até certa medida, concentrar-se no processo de aprendizado, no domínio do conteúdo e de realização de tarefa, características da meta aprender (Senko \& Hulleman, 2013), pode explicar aspectos como um maior planejamento, monitoramento, regulação do próprio pensamento e manutenção de um estado interno satisfatório à aprendizagem do indivíduo, o que corresponde às estratégias metacognitivas (Dembo, 1994; Scacchetti et al., 2015).

Por fim, as metas aprender e performance-evitação foram consideradas preditoras das estratégias de aprendizagem. Com isso, verifica-se que a preocupação 
com a aprendizagem por si mesma e pela evitação de desvalorização entre os colegas, professores etc. (Bzuneck \& Boruchovitch, 2016; Zenorini \& Santos, 2010) pode explicar o emprego de estratégias de aprendizagem no momento de aprender, já que estas contribuem não somente para esse fim, mas também para um melhor desempenho acadêmico (Prates et al., 2016; Zenorini \& Santos, 2010). Cabe ressaltar que não foram encontradas na literatura pesquisas que houvessem realizado verificações de predição entre os construtos da motivação e das estratégias de aprendizagem, o que dificulta dados de comparação dos resultados e reforça a importância do presente estudo.

\section{Considerações finais}

É possível considerar que o presente estudo atingiu os objetivos aos quais se propôs, já que os dados evidenciaram a tendência dos alunos do ensino médio em pontuar mais expressivamente os itens da meta aprender e o emprego de estratégias metacognitivas no momento da aprendizagem. Esses resultados podem ser considerados positivos à medida que contribuem para o sucesso acadêmico do estudante. Os achados também foram parcialmente congruentes com outras pesquisas nacionais, visto que existe incipiência de estudos acerca da temática em questão.

Evidencia-se a relevância do estudo à medida que auxilia na ampliação da compreensão dos processos de aprendizagem em alunos no ensino médio e na análise dos diversos fatores interligados à questão do aprender a aprender. Não obstante isso, ressalta-se que este estudo não foi empreendido sem limitações, que devem ser mencionadas para que possam nortear a continuidade das investigações realizadas. Pode-se citar a singularidade da amostra, quer por ser proveniente de uma única cidade do Nordeste (Salvador/BA), quer por pertencer a uma instituição particular de ensino. Outra limitação refere-se ao uso de uma escala empreendida e com propriedades psicométricas para o ensino fundamental, pois, mesmo tendo sido realizada uma análise semântica dos itens antes de sua aplicação, não participaram juízes especialistas e não houve um estudo-piloto com estudantes do ensino médio realizado anteriormente.

Assim, novas pesquisas são requeridas de forma a investigar mais amplamente os construtos da motivação e das estratégias de aprendizagem, bem como ressalta-se a importância de que outras variáveis psicoeducacionais, como a autoeficácia acadêmica e a autorregulação da aprendizagem, sejam analisadas conjunta- 
mente. Por fim, propõe-se também, como agenda futura de pesquisa, a elaboração ou adaptação de um instrumento relativo às estratégias de aprendizagem para o ensino médio brasileiro.

\section{Referências}

Albuquerque, E. A., Batista, F. F., Albuquerque, L. S., Oliveira, F. D. C., \& Pereira, G. M. (2016). Análise da motivação dos discente do curso de ciências contábeis e administração sob a perspectiva da teoria da autodeterminação e das metas de realização. Qualitas, 17(3), 1-21. Recuperado de http://revista.uepb.edu.br/index.php/ qualitas/article/view/3043/pdf

Alcará, A. R., \& Santos, A. A. A. (2013). Compreensão de Leitura, estratégias de aprendizagem e motivação em universitários. Psico, 44(3), 411-420. Recuperado de http:// revistaseletronicas.pucrs.br/ojs/index.php/revistapsico/article/view/12258/10416

Beluce, A. C., \& Oliveira, K. L. O. (2016). Escala de estratégias e motivação para aprendizagem em ambientes virtuais. Revista Brasileira de Educação, 21(66), 593-610. doi:10.1590/S1413-24782016216631

Bzuneck, J. A., \& Boruchovitch, E. (2016). Motivação e autorregulação da motivação no contexto educativo. Psicologia: Ensino \& Formação, 7(2), 73-84. doi:10.21826/217958002016727584

Castro, J. X., Miranda, G. J., \& Leal. E. A. (2016). Estratégias de aprendizagem dos estudantes motivados. Advances in Scientific and Applied Accounting, 9(1), 80-97. Recuperado de http://asaa.anpcont.org.br/index.php/asaa/article/view/258

Cohen, J. (1988). Statistical power analysis for the behaviral sciences. Hillsdale, NJ: Erlbaum.

Costa, E. R., \& Boruchovitch, E. (2015). O ensino de estratégias de aprendizagem no contexto da escrita. Psicologia da Educação, (41), 21-35. doi:10.5935/21753520.20150012

Dal'Igna, M. C. (2007). Desempenho escolar de meninos e meninas: Há diferença? Educação em Revista, 46, 241-267. doi:10.1590/S0102-46982007000200010

Dembo, M. H. (1994). Applying educational psychology (5th ed.). New York: Longman.

Elliot, A. J., \& McGregor, H. A. (2001). A $2 \times 2$ achievement goal framework. Journal of Personality and Social Psychology, 8o(3), 501-519. doi:10.1037/0022-3514.80.3.501

Elliot, A. J., \& Murayama, K. (2008). On the measurement of achievement goals: Critique, illustration, and application. Journal of Educational Psychology, 100(3), 613628. doi:10.1037/0022-0663.100.3.613 
Elliott, E. S., \& Dweck, C. S. (1988). Goals: An approach to motivation and achievement. Journal of Personality and Social Psychology, 54(1), 5-12. Recuperado de https://www. ncbi.nlm.nih.gov/pubmed/3346808

Ferraz, A. S., Cantalice, L. M., \& Santos, A. A. A. (2019). Motivação para aprender e compreensão de leitura em alunos do ensino fundamental I. Estudos Interdisciplinares em Psicologia, 10(1), 173-189. doi:10.5433/2236-6407.2019v10n1p173

Ferreira, E. B., \& Silva, M. R. (2017). Centralidade do ensino médio no contexto da nova "ordem e progresso". Educação \& Sociedade, 38(139), 287-292. doi:10.1590/ es0101-73302017179021

Greene, B. A., Miller, R. B., Crowson, H. M., Duke, B. L., \& Akey, K. L. (2004). Predicting high school students' cognitive engagement and achievement: Contributions of classroom perceptions and motivation. Contemporary Educational Psychology, 29(4), 462-482. doi:10.1016/j.cedpsych.2004.01.006

Inácio, A. L. M. (2018). Estilos intelectuais, estratégias de aprendizagem, compreensão de leitura e desempenho escolar no ensino médio. Dissertação de mestrado, Universidade Estadual de Londrina, Londrina, PR, Brasil.

Krawczyk, N. (2011). Reflexões sobre alguns desafios do ensino médio no Brasil hoje. Cadernos de Pesquisa, 41(144), 752-769. doi:10.1590/S0100-15742011000300006

Lemos, G. C., Almeida, L. S., Guisande, C. M. A., Barca, L. A., Primi, R., Martinho, G., \& Fortes, I. (2010). Inteligência e rendimento escolar: Contingências de um relacionamento menos óbvio ao final da adolescência. Revista Galego-Portuguesa de Psicoloxía e Educacíon, 18(1), 163-175. Recuperado de https://ruc.udc.es/dspace/ handle/2183/8397

Lima, T. H., \& Santos, A. A. A. (2016). Compreensão de leitura e uso de estratégias de aprendizagem: Estudo correlacional. Argumentos Pró-Educação, 1(1), 103-117. Recuperado de http://ojs.univas.edu.br/index.php?journal=argumentosproeducacao\& page=article\&op=view \&path\%5B\%5D=64\&path\%5B\%5D $=77$

Maieski, S., Oliveira, K. L., Beluce, A. C., \& Rufini, S. E. (2017). Motivação de alunos do ensino fundamental: Estudo de duas realidades culturais. Psicologia Escolar e Educacional, 21(3), 601-608. doi:10.1590/2175-353920170213111129

Oliveira, K. L., Boruchovitch, E., \& Santos, A. A. A. (2010). Escala de Avaliação das Estratégias de Aprendizagem para o Ensino Fundamental (EAVAP-EF). São Paulo: Casa do Psicólogo.

Oliveira, K. L., Boruchovitch, E., \& Santos, A. A. A. (2011). Estratégias de aprendizagem no ensino fundamental: Análise por gênero, série escolar e idade. Psico, 42(1), 98- 
105. Recuperado de http://revistaseletronicas.pucrs.br/ojs/index.php/revistapsico/ article/view/6273/6305

Oliveira, K. L., Santos, A. A. A., \& Inácio, A. L. M. (2017). Estratégias de aprendizagem no ensino médio brasileiro: Uma análise exploratória dos resultados. Revista de Estudios e Investigación en Psicología y Educación, Extr.(1), 337-341. doi:10.17979/ reipe.2017.0.01.3041

Perassinoto, M. G. M., Boruchovitch, E., \& Bzuneck, J. A. (2013). Estratégias de aprendizagem e motivação para aprender de alunos do ensino fundamental. Avaliação Psicológica, 12(3), 351-359. Recuperado de http://pepsic.bvsalud.org/scielo. php?script=sci_arttext\&pid=S1677-04712013000300010

Prates, K. C. R., Lima, R. F., \& Ciasca, S. M. (2016). Estratégias de aprendizagem e sua relação com o desempenho escolar em crianças do ensino fundamental I. Revista Psicopedagogia, 33(100), 19-27. Recuperado de http://pepsic.bvsalud.org/pdf/psicoped/v33n100/03.pdf

Santos, A. A. A., Moraes, M. S., \& Lima, T. H. (2018). Compreensão de leitura e motivação para aprendizagem de alunos do ensino fundamental. Psicologia Escolar e Educacional, 22(1), 93-101. doi:10.1590/2175-35392018012208

Scacchetti, F. A. P., Oliveira, K. L., \& Moreira, A. E. C. (2015). Estratégias de aprendizagem no ensino técnico profissional. Psico-USF, 20(3), 433-446. doi:10.1590/1413-82712015200306

Senko, C., \& Hulleman, C. S. (2013). The role of goal attainment expectancies in achievement goal pursuit. Journal of Educational Psychology, 105(2), 504-521. doi:10.1037/aoo3/136.

Suehiro, A. C. B., Boruchovitch, E., \& Schelini, P. W. (2018). Estratégias de aprendizagem e a regulação da emoção no ensino fundamental. Estudos Interdisciplinares em Psicologia, 9(3), 90-111. Recuperado de http://pepsic.bvsalud.org/scielo.php?script= sci_arttext\&pid=S2236-64072018000400007

Weinstein, C. E., \& Mayer, R. E. (1983). The teaching of learning strategies. Innovation Abstracts, 5(32), 1-4. Recuperado de http://files.eric.ed.gov/fulltext/ED237180.pdf

Zenorini, R. P. C., \& Santos, A. A. A. (2010). Escala de metas de realização como medida da motivação para aprendizagem. Revista Internacional de Psicologia, 44(2), 291298. Recuperado de https://www.redalyc.org/html/284/28420641010/ 


\section{Nota dos autores}

Acácia Aparecida A. dos Santos, Programa de Pós-Graduação Stricto Sensu em Psicologia, Universidade São Francisco (USF); Amanda Lays M. Inácio, Programa de Pós-Graduação Stricto Sensu em Psicologia, Universidade São Francisco (USF).

Correspondências referentes a este artigo devem ser encaminhadas para Amanda Lays Monteiro Inácio, Departamento de Psicologia e Psicanálise, Centro de Ciências Biológicas, Universidade Estadual de Londrina, Campus Universitário, Rodovia Celso Garcia Cid (PR 445), km 380, Londrina, PR, Brasil. Caixa Postal 6001. CEP 86051-990.

E-mail: amandalmonteiroo@gmail.com 